\title{
Intervención Cognitivo-Conductual en Jugadores Mexicanos de Fútbol Profesional. Cognitive- Behavioral Intervention in Mexican Professional Soccer Players
}

\author{
Hebert Mercado Guerrero, Licenciado \\ Rebeca María Elena Guzmán Saldaña, Doctora \\ Universidad Autónoma del Estado de Hidalgo. \\ Instituto de Ciencias de la Salud. Área Académica de Psicología. \\ Laszlo Egry Bulnes, Maestro \\ Centros de Desarrollo Humano del Sistema DIF del Estado de Hidalgo, \\ México

\section{Arturo del Castillo Arreola, Doctor} \\ Angélica Romero Palencia, Doctora \\ Universidad Autónoma del Estado de Hidalgo. \\ Instituto de Ciencias de la Salud. Área Académica de Psicología.
}

\begin{abstract}
This pre-experimental study aimed to analyze the effects of a training program to improve athletic performance in soccer using cognitivebehavioral techniques during 12 sessions. The study involved 18 men professional players, with an average age of 19.50 years $(D E=1.42)$. Athletic performance and the intervention included variables such as motivation, stress management, performance appraisal influence, mental ability and group cohesion that were evaluated through two applications (pre and post) of the "Psychological Characteristics of Sport Performance Questionnaire" (CPRD). A first analysis of the data showed no significant group differences between times of measurement; however, the Wilcoxon test yielded data that showed intra-individual changes in the variables mental ability and group cohesion. It's important to point out that, the team won the championship after their participation in the intervention. The study reveals the importance of the psychologist work in the sports field.
\end{abstract}

Keywords: Sports Performance, Cognitive-Behavioral Intervention, Professional Soccer Players 


\section{Resumen}

Este estudio tuvo como objetivo analizar los efectos de un programa de entrenamiento para mejorar el rendimiento deportivo en fútbol usando técnicas cognitivo-conductuales durante 12 sesiones. El diseño fue preexperimental, participaron $\mathrm{N}=18$ hombres, jugadores profesionales, con una media de edad de 19.50 años $(D E=1.42)$. El rendimiento deportivo y la intervención consideró variables como: motivación, control de estrés, influencia de evaluación del rendimiento, habilidad mental y cohesión grupal, que se evaluaron a través de dos aplicaciones (pre y post) del Cuestionario de Características Psicológicas para el Rendimiento Deportivo (CPRD). Un primer análisis de datos grupal no mostró diferencias significativas entre los distintos momentos de medición; sin embargo, la prueba de Wilcoxon arrojó resultados que evidenciaron cambios intraindividuales en las variables: habilidad mental y cohesión grupal. Es importante destacar que después de la participación en la intervención el equipo obtuvo el Campeonato. El estudio revela la importancia de la labor del psicólogo dentro del campo del deporte.

Palabras Clave: Rendimiento Deportivo, Intervención CognitivoConductual, Fútbol Soccer, Jóvenes.

\section{Introducción}

El rendimiento de un deportista depende de una serie de factores que se integran en grandes categorías que le influyen directamente. Entre otros, están los considerados más importantes como: la condición física, los aspectos técnico-tácticos y estratégicos, los materiales o implementos deportivos, los factores externos dentro de la competición y las habilidades psicológicas; sin dejar de lado los factores del ambiente que repercuten directamente en algunas de estas categorías (González Campos, Campos Mesa, \& Romero Granados, 2014).

En cualquier deporte la necesidad de generar intervenciones efectivas para potenciar los recursos psicológicos tanto individuales como grupales cobra relevancia, sobre todo en aquellos en los que el resultado final depende de la interacción eficaz entre sus miembros, de tal forma que se logre un óptimo rendimiento que se refleje en un buen resultado (e. g. Carmona, Guzmán, \& Olmedilla, 2015; Castillo-Rodríguez, 2011; Chicau, Silva, \& Palmi, 2013; Lorenzo, Pujals, Navarro, \& Lorenzo, 2012; Sánchez-Sánchez, Yagüe, \& Molinero, 2013; Serra-Olivares, González-Villora, \& GarcíaLópez, 2011).

Por ejemplo, el fútbol pertenece al grupo de deportes de colaboración-oposición, siendo cada jugador una parte integrada al equipo; en donde regularmente de manera personal al individuo por sí mismo no se 
le evalúa (necesariamente), ya que lo que cuenta es el resultado final como equipo (Boillos, 2006).

El profesional de la psicología debe trabajar en sentido amplio, para que los deportistas mejoren el dominio de las habilidades psicológicas que requiere cada especialidad deportiva, desde el uso de técnicas y estrategias psicológicas, hasta los procesos de reflexión y toma de decisiones, tanto en el ámbito deportivo como extra deportivo (Olmedilla, Ortega, Ortín, \& Andreu, 2008).

Diversos estudios han reportado el papel que juegan elementos como la motivación, la cohesión de equipo y las tendencias de comportamiento sobre el desempeño atlético, y de una manera central la forma en que la atención y el control del estrés afectan el rendimiento deportivo (e. g. Bara, Andrade, Miranda, Núñez, Martín-Albó, \& Ribas, 2011; Burtscher, Furtner, Sachse, \& Burtscher, 2011; Cerasoli, Nicklin, \& Ford, 2014; GraneroGallegos \& Baena-Extremera, 2013; Perry, 2015).

La motivación, la atención, la autoconfianza, el control del estrés o, de forma general, el autocontrol o autorregulación emocional y las habilidades interpersonales, representan, tal y como han señalado diversos expertos, las claves para entender las diferencias de nivel mostradas por los deportistas. Conocer la repercusión de estas variables constituye el primer paso en el proceso de formación deportiva y por lo tanto debe ser previo a cualquier tipo de intervención psicológica (López-López, Jaenes-Sánchez, \& Cárdenas-Vélez, 2013).

El entrenamiento psicológico según García-Ucha (2009) consiste en actividades dirigidas al deportista para la adquisición y desarrollo de habilidades que faciliten el accionar más eficiente, y tiene como objetivos mejorar la capacidad de autorregulación, enfrentar el estrés en competencias $\mathrm{y}$ entrenamientos, perfeccionar las capacidades en el deporte y en la competición, y finalmente preparase para reorganizar los recursos psicológicos ampliando la gama de posibilidades mediante el afrontamiento de situaciones nuevas e imprevistas. Implica además el desarrollo personal y profesional y la mejora del desempeño de directores deportivos, entrenadores y deportistas (García Naveira, 2011).

Menéndez-Veliz y Estua-Ampudia (2013), precisan sobre la adquisición de habilidades mentales para incrementar el rendimiento, así como el crecimiento humano, específicamente en cuanto a los motivos deportivos, el desarrollo de las cualidades volitivas, y el perfeccionamiento de las particularidades del pensamiento táctico. Estos elementos también potencian la ejecución en entrenamientos y competiciones, así mismo, en períodos de reposo y recuperación, y en el establecimiento de rutinas o planes de acción dirigidos a organizar la actuación del deportista antes, 
durante y después de las competiciones poniendo en práctica las habilidades psicológicas aprendidas (Godoy-Izquierdo, Vélez, \& Pradas, 2009).

Ahora bien, desde la perspectiva psicológica el enfoque cognitivoconductual de entrenamiento deportivo, toma en cuenta tanto factores externos al individuo como internos; particularmente la aplicación de técnicas tiene como objetivo principal ayudar a la persona a desarrollar un conjunto de herramientas útiles para manejar y/o superar problemas a nivel de las cogniciones (pensamientos, creencias y esquemas mentales), que conlleve a la disminución y/o desaparición de emociones perturbadoras (e.g. ansiedad), y de comportamientos desadaptativos (e. g. compulsiones, conductas de escape y evitación, entre otras) (Boot, Simons, \& Stothart, 2013).

El desarrollo de situaciones estresantes durante las competiciones deportivas implica en muchas ocasiones alteraciones del funcionamiento psicológico, tales como una menor capacidad de concentración, una pérdida del foco atencional, un aumento de la ansiedad-estado o, desde el punto de vista somático, un incremento de la tensión muscular (Brown \& Fletcher, 2016; McCormick, Meijen, \& Marcora,2015; Rumbold, Fletcher, \& Daniels, 2012; Wagstaff, 2014).

El estrés se presenta cuando un individuo se ve amenazado de no cumplir con sus capacidades en una situación y que puede tener consecuencias físicas (como lesiones y dolencias), psicológicas (e. g. problemas emocionales, altos niveles de ansiedad, bajos niveles de motivación y altas expectativas) y sociales (e. g. falta de cohesión grupal, huida, abandono y aislamiento) (Garcés de Los Fayos, De Francisco, \& Arce, 2012). Sin lugar a dudas, un jugador de fútbol se enfrenta a una carrera deportiva muy competitiva en la que deberá aprender a soportar situaciones potencialmente estresantes (Boillos, 2006; Fenoy \& Campoy, 2012; González-Campos, Valdivia-Moral, Zagalaz, \& Romero, 2015).

Así mismo, el factor de habilidad mental se relaciona con las representaciones de movimiento en el cerebro del deportista que si se llevan a cabo en forma inexacta, errónea o inadecuada, la ejecución será también incorrecta (Carmona, Guzmán, \& Olmedilla, 2015).

El objetivo final del entrenamiento psicológico es enseñar al deportista a encontrar un estado mental ideal (García \& Serrano, 2011; Godoy-Izquierdo et. al, 2009) en el que las variables psicológicas relevantes para el aprendizaje y la ejecución de las destrezas deportivas se encuentren en sus niveles óptimos de cara a incrementar el rendimiento del deportista, así como el alcanzar, mantener o potenciar ese estado y lograr un rendimiento superior; especialmente en la competición, mediante la autoaplicación de las habilidades psicológicas autorreguladoras entrenadas 
(González-Campos, 2011; Lorenzo \& Bohorquez, 2014; Pazo, Sáenz-López, \& Fradua, 2012).

Para explicar los potenciales efectos negativos de la ansiedad en el rendimiento deportivo, se plantean tres mecanismos o factores principales: a) Perturbación o desvío atencional hacia pensamientos irrelevantes y/o conflicto motivador, b) "Déficit” de capacidades o competencias físicas, tácticas, técnicas u otras, lo que presupone que pueden ser también los débiles rendimientos anteriores que desencadenen la respuesta ansiosa y no el inverso, y c) Procesos de enfrentamiento defensivos que tratan de proteger el autoestima del atleta, llevándolo a no desarrollar todo el esfuerzo y persistencia en la ejecución de la actividad (García, 2008; García \& Serrano, 2011; López-López, 2011; Navlet, 2012).

A diferencia de lo que sucede en otras modalidades consideradas de dominancia energético funcional, o de carácter técnico combinado, en el fútbol los componentes táctico y psicológico parecen condicionar de forma importante el rendimiento del jugador (Cachón, Rodrigo, Campoy, Linares, \& Zagalaz, 2012).

Cobra relevancia la cohesión grupal (que corresponde a la medida de fuerza que mantiene unido a un grupo) para lograr un mejor rendimiento del equipo (Eys, Loughead, Bray, \& Carron, 2009). Específicamente ese término hace referencia a dos dimensiones: cohesión de tarea, que refleja el grado en que los miembros del grupo trabajan juntos para alcanzar objetivos comunes, y la cohesión social que son los lazos de atracción interpersonal que ligan a los miembros del grupo (Carron, Colman, Wheeler, \& Stevens, 2002; Carron, Hausenblas, \& Eys, 2005). El modelo conceptual de cohesión de Carron (1998) propone cuatro antecedentes principales que afectan al desarrollo de la cohesión en el ámbito del deporte y el ejercicio físico: factores ambientales, factores personales, factores de liderazgo y factores de equipo, relacionándose así con factores motivacionales y de liderazgo como el clima motivacional, las orientaciones o el tipo de motivación (GarcíaCalvo, Leo-Marcos, Sánchez-Miguel, Jiménez-Casturera, \& CervellóGimeno, 2008).

Por otro lado, el uso de técnicas cognoscitivas como la visualización mantiene el control de las emociones y del cuerpo (McWhirter, 2016). La visualización o imaginería es el proceso mediante el cual se pueden crear, modificar y fortalecer circuitos esenciales para la coordinación de los músculos del deportista, sólo entrenando la mente. La activación mental de una acción física implica que el sistema neuronal de todo organismo se "encienda" como si los músculos acompañaran a la vivencia. Imaginería es entrenamiento sin gasto de energía. Los atletas que la utilizan tienen menos accidentes, menos dolor, menor tensión muscular, un sueño adecuado y reparador; y cuando la técnica se automatiza, los niveles de auto-confianza 
se incrementan (Álvarez, Falco, Estevan, Molina-García, \& Castillo, 2013).

La relajación además de producir sensaciones subjetivas de bienestar, también produce actividad en los músculos; por lo tanto, favorece los mecanismos fisiológicos necesarios para mantener esta actividad muscular (Cantón \& Checa, 2011). Las técnicas respiratorias mejoran la oxigenación, el tono muscular y garantizan en general una vida más sana, en los deportistas los malos hábitos de respiración ocasionan esfuerzos extraordinarios (Jouper \& Gustafsson, 2013; Nieuwenhuys \& Oudejans, 2011).

El objetivo del presente estudio fue evaluar el efecto de una intervención cognitivo conductual para mejorar el rendimiento deportivo en fútbol, usando técnicas cognitivo-conductuales durante doce sesiones, en jugadores profesionales. Cabe señalar, que cada semana de trabajo tenía uno o varios objetivos, cada uno de ellos relacionado con el entrenamiento grupal en técnicas de motivación, relajación a través de la respiración, la visualización, y finalmente el trabajo con la cohesión grupal.

\section{Método}

\section{Participantes}

En el estudio participaron $\mathrm{N}=18$ jugadores de un equipo de fútbol de segunda división profesional, que en promedio de edad tenían 19.50 años $(D E=1.42)$.

InstrumentosPara evaluar el rendimiento deportivo se administró el Cuestionario de Características Psicológicas para el Rendimiento Deportivo (CPRD) de Gimeno, Buceta y Pérez Llantada, (2001) en la versión adaptada para México (Ramírez, 2001).

El CPRD es un Inventario tipo Likert, auto aplicable de lápiz y papel, que consta de 55 reactivos, que evalúan cinco factores: motivación, control de estrés, influencia de evaluación del rendimiento, habilidad mental y cohesión grupal.

\section{Procedimiento}

Como ya se señaló, el estudio se desarrolló en doce sesiones interactivas-vivenciales, con enfoque cognitivo-conductual, diseñado ex profeso, cuyas temáticas de entrenamiento en los jugadores fueron: motivación, relajación a través de la respiración, la visualización y cohesión grupal.

Se inició con la invitación y obtención del consentimiento informado para participar, dirigida a las autoridades y jugadores del equipo de fútbol; posteriormente de forma individual se realizó la pre evaluación con la aplicación del CPRD, después se llevaron a cabo las sesiones, de manera 
grupal, dos veces por semana, con una duración aproximada de una hora. El lugar de la intervención variaba de acuerdo con las actividades programadas para el entrenamiento del equipo, dentro de los espacios que se utilizaban para la intervención se encuentran un gimnasio y un salón de unidad deportiva. Al finalizar la intervención se aplicó nuevamente el CPRD.

\section{Análisis de datos}

Para la realización de este estudio se utilizó el programa SPSS versión 20.0. Al realizar el análisis estadístico, se observó mediante la prueba de Shapiro-Wilks que las puntuaciones psicológicas de los jugadores y la variable de rendimiento deportivo no se distribuían de forma normal, por lo que se utilizó estadística no paramétrica.

Para analizar las diferencias en el rendimiento deportivo se utilizó el estadístico W de Wilcoxon, que permitió comparar las dos medidas intrasujeto, una tomada antes de la intervención y otra al finalizar ésta.

Respecto al análisis de las variables de rendimiento, y debido a que las variables se midieron en partidos de fútbol diferentes, se realizaron pruebas U de Mann-Whitney para analizar las diferencias en el rendimiento en los partidos analizados en función de la intervención (pre y posttratamiento).

\section{Resultados}

Para conocer los cambios de los participantes en cada una de las subescalas del CPRD, como ya señaló se muestran los resultados en cada participante de acuerdo con el análisis de rangos de Wilcoxon.

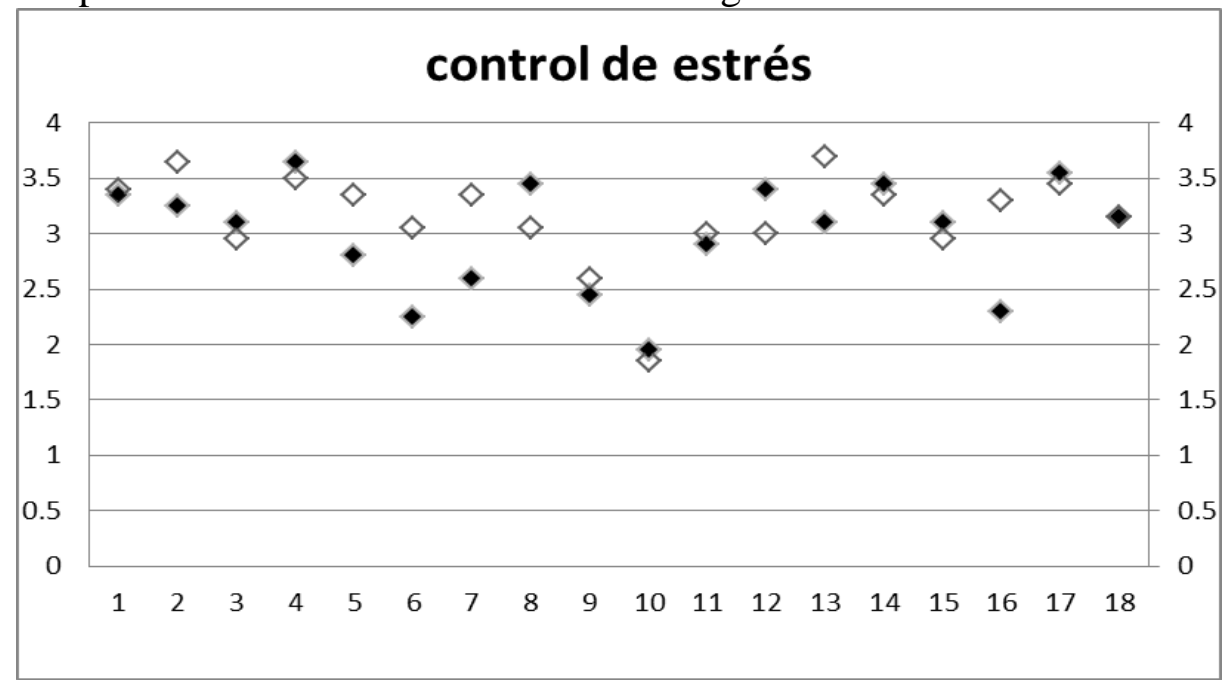

Nota. Rombos Blancos=Antes de la intervención; Rombos Negros= Después de la intervención.

Figura 1. Cambios de cada participante en el análisis de rangos de Wilcoxon en el factor de control de estrés. 
Al realizar la comparación pre-post evaluación respecto al factor control de estrés, se puede observar en la figura 1 , que en total $n=8$ de los participantes mejoraron el manejo del estrés $(3=.60,4=.60,8=.40,10=.10$, $12=.40,14=.10,15=.60,17=.10)$; en cambio $\mathrm{n}=7$ lo disminuyeron $(2=-.40$, $5=-.55 ; 6=-.80,7=-.75,9=-.60,13=-.60,16=-.10)$, $\mathrm{y}$ por último $\mathrm{n}=3 \mathrm{de}$ los participantes no tuvieron cambios (1,11 y 18$)$.

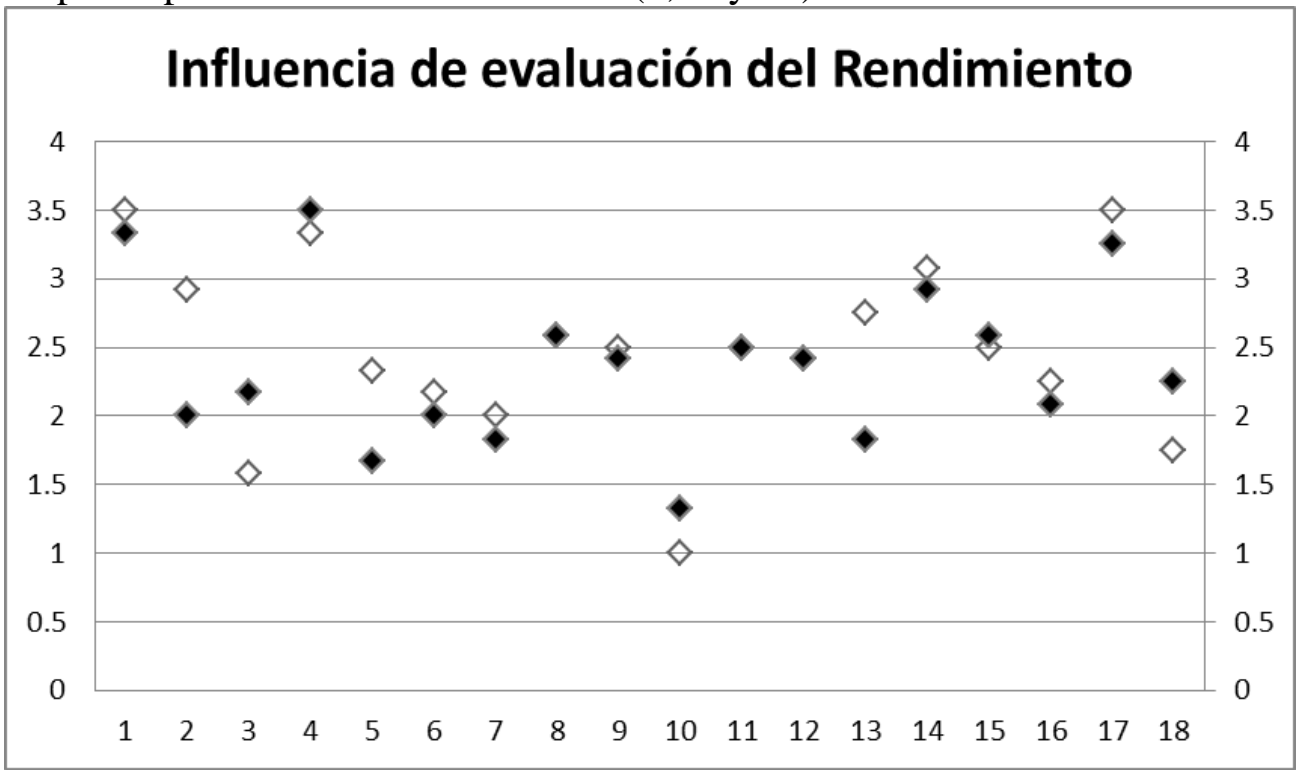

Nota: Rombos Blancos= antes de la intervención; Rombos Negros= después de la intervención.

Figura 2. Cambios de cada participante en el análisis de rangos de Wilcoxon en el factor influencia de evaluación del rendimiento.

Como puede observarse en la figura 2, respecto al factor de Influencia de evaluación del rendimiento fueron $\mathrm{n}=4$ jugadores los que obtuvieron resultados favorables $(3=.59,4=.17,10=.33,18=.50)$; por el contrario, $n=9$ de los participantes manifestaron evaluaciones desfavorables $(1=-.17,2=-.92$, $5=-.66,6=-.17,7=-.17,13=-.92,14=-.16,16=-.17,17=-.25), \mathrm{y} \mathrm{n}=5 \mathrm{no}$ presentaron cambios $(8,9,11,12$ y 15).

En el factor Motivación, $\mathrm{n}=6$ de los participantes mejoraron $(1=.25$, $3=.25,4=.13,9=.13,12=.26,16=.12) ; \mathrm{n}=9$ disminuyeron sus puntajes $(2=-$ $.25,5=-.12,6=-.37,7=-.38,10=-.64,13=-.25,15=-.37,17=-.12,18=-$ .38 ), $y$ en $n=3$ jugadores no se modificaron sus puntajes $(8,11,14)$ (ver figura 3). 


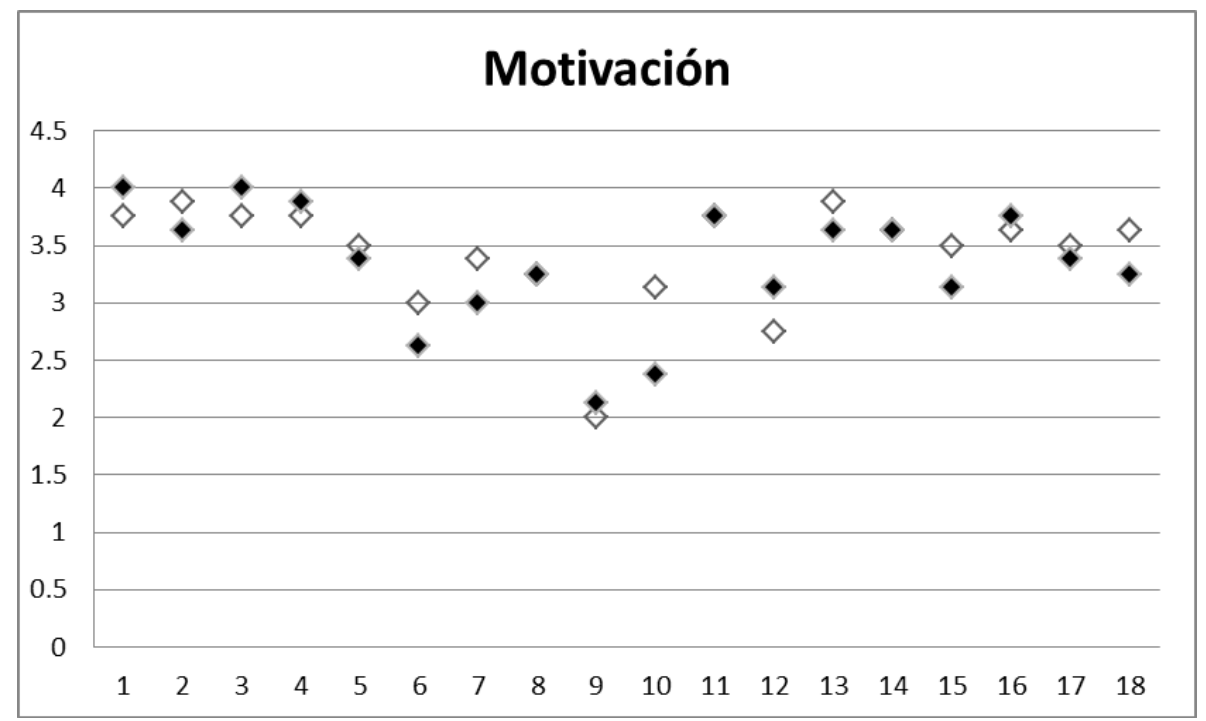

Nota: Rombos Blancos= antes de la intervención; Rombos Negros= después de la intervención.

Figura 3. Cambios de cada participante en el análisis de rangos de Wilcoxon en el factor de Motivación.

En la figura 4 se aprecia que en el factor de habilidad mental más de la mitad de los jugares mejoraron de manera importante sus puntajes en la post evaluación $(2=.22,3=.11,4=.11,8=.57,9=.22,11=.40,12=.67,13=$ $.44,14=.55,16=.12,18=.89) ; \mathrm{n}=6$ disminuyeron $(1=-.44,5=-.11,6=-.11$, $7=-.55,10=-.34,15=-.57)$, y un participante no tuvo cambios (17).

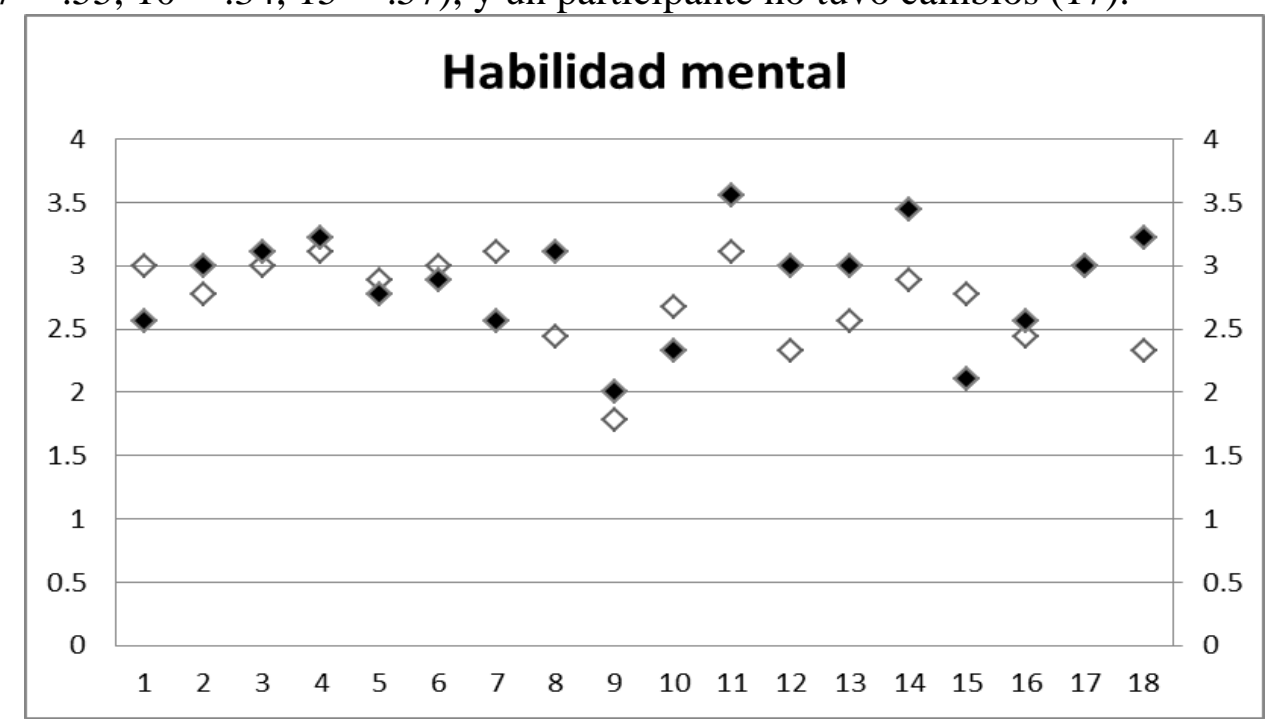

Nota: Rombos Blancos= antes de la intervención; Rombos Negros= después de la intervención.

Figura 4. Cambios de cada participante en el análisis de rangos de Wilcoxon en el factor de habilidad mental. 
Finalmente en el factor Cohesión Grupal, $\mathrm{n}=7$ de los participantes mejoraron sus puntajes $(1=.17,2=.17,11=.13,12=.67,14=.50,15=.33$, $18=.33), \mathrm{n}=5$ los disminuyeron $(5=-.34, \quad 7=-.66,8=-.66,13=-.66$, $16=-.12)$, y por último, $\mathrm{n}=6$ no mostraron cambios $(3,4,6,9,10,17)$ (ver figura 5).

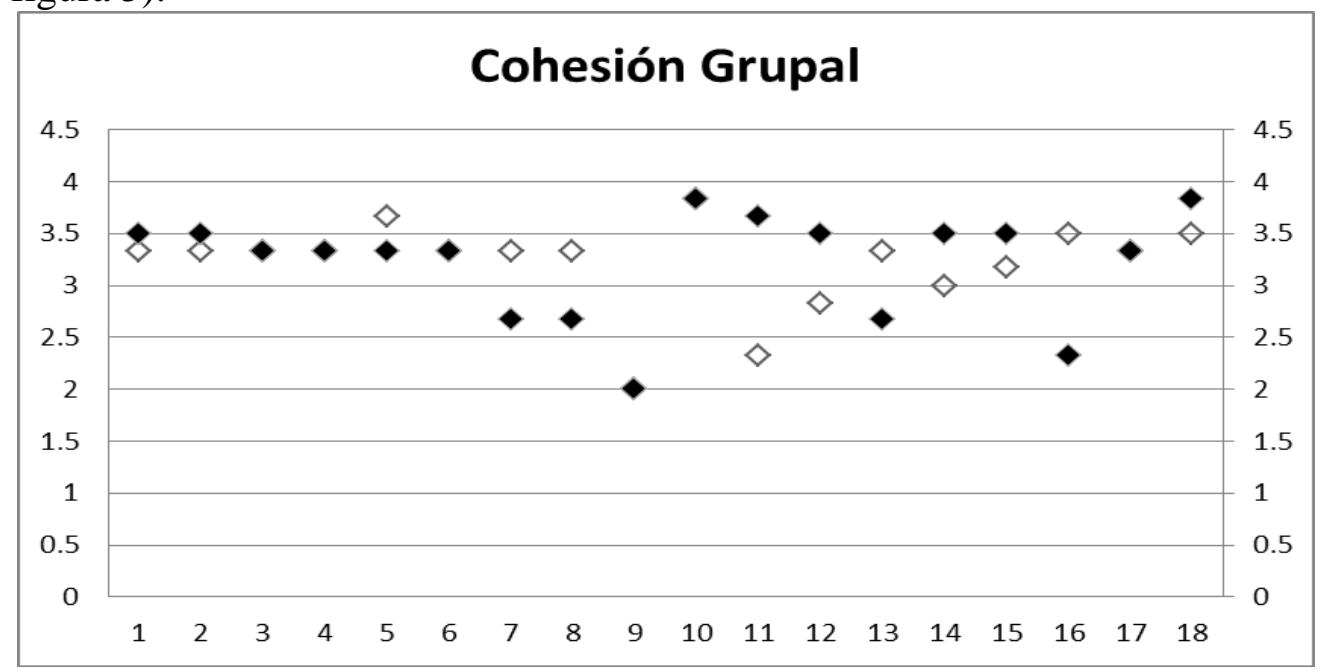

Nota: Rombos Blancos= antes de la intervención; Rombos Negros= después de la intervención.

Figura 5. Cambios de cada participante en el análisis de rangos de Wilcoxon en el factor de cohesión grupal.

DiscusiónSin lugar a dudas el rendimiento deportivo está condicionado por tres tipos de preparación: física, técnico-táctica, y psicológica, si alguna de estas se descuida es probable que el rendimiento se vea disminuido. En este sentido el desarrollar intervenciones psicológicas que contemplen técnicas de motivación, relajación a través de la respiración, la visualización, y trabajo con la cohesión grupal entre otras cobra relevancia.

Por tal motivo, el propósito del presente estudio fue analizar los efectos de un programa de entrenamiento psicológico usando técnicas cognitivo-conductuales para mejorar el rendimiento deportivo aplicado en jugadores en fútbol de la segunda división profesional.

Los datos arrojados en donde se aplicó el Inventario CPRD se analizaron a través de la prueba estadística de Wilcoxon con la finalidad de identificar los cambios en la pre y post evaluación que presentaron de manera individual 18 jugadores profesionales participantes en este estudio.

Es interesante destacar que los mejores resultados se obtuvieron en el factor de habilidad mental, siguiéndole el factor cohesión grupal y después control de estrés, a continuación se discute al respecto. 
El factor de habilidad mental está relacionado con las representaciones de movimientos tácticos que el deportista realiza en su cerebro, y que si se llevan a cabo en forma inexacta, errónea o inadecuada, la ejecución será también incorrecta (Carmona, Guzmán, \& Olmedilla, 2015); de ahí que el objetivo del entrenamiento psicológico en este factor mostró evidencias de cambios favorables que permitieron a los futbolistas encontrar un estado mental ideal.

Estos datos son semejantes a los encontrados por Godoy-Izquierdo, Vélez y Pradas (2009) en un estudio en donde afirmaron que variables psicológicas como la habilidad mental es relevante para el aprendizaje, así como la ejecución de las destrezas deportivas, de tal forma que se mantenga en niveles óptimos, y que esto se refleje en un incremento en el rendimiento deportivo del jugador, especialmente en la competición. En este sentido, se puede afirmar que se lograron resultados positivos al estimular en los jugadores la capacidad que tienen las personas desde la niñez de visualizar, pero que desafortunadamente se va perdiendo al ir entrando en el mundo de los adultos, ya que la cultura exige cada vez más practicar las capacidades analíticas, y dejar a un lado las de imaginación (García \& Serrano, 2011; Gimeno, Buceta \& Pérez-Llantada, 2001; Godoy-Izquierdo et. al, 2009; McWhirter, 2016).

Así mismo, la cohesión grupal mostró diferencias significativas en los dos momentos de evaluación; que en distintos momentos (incluyendo el logro del Campeonato de Liga) permitió observar a un grupo unido, con un adecuado rendimiento como equipo. La cohesión mostró evidencias en cuanto a la realización de la tarea, en donde los jugadores del equipo trabajaron juntos para alcanzar objetivos comunes; además, se manifestó la cohesión social estableciendo lazos adecuados de relaciones interpersonales.

A través de los resultados de este estudio se pudieron confirmar los elementos que Carron (1998) propone como antecedentes principales de la cohesión grupal en el ámbito del deporte y el ejercicio físico, tales como factores ambientales, factores personales, factores de liderazgo y factores de equipo, asociándose con factores motivacionales y de liderazgo como el clima motivacional, las orientaciones o el tipo de motivación (Carron, Colman, Wheeler, \& Stevens, 2002; Carron, Hausenblas, \& Eys, 2005; García-Calvo, Leo-Marcos, Sánchez-Miguel, Jiménez-Casturera, \& Cervelló-Gimeno, 2008).

En cuanto al factor control de estrés también se obtuvieron adecuados puntajes, aún y cuando los jugadores tuvieron que competir y ganar el Campeonato de esa temporada en la que se realizó el estudio. Es importante señalar que, si una persona se ve amenazada por no cumplir con sus capacidades en una situación determinada, incrementa la posibilidad de tener consecuencias físicas (como lesiones), psicológicas (e. g. problemas 
emocionales, altos niveles de ansiedad, bajos niveles de motivación y altas expectativas) y sociales (e. g. falta de cohesión grupal, huida, abandono y aislamiento) (Carmona, Guzmán, \& Olmedilla, 2015). Un jugador de fútbol se enfrenta a una carrera deportiva muy competitiva en la que deberá aprender a soportar situaciones potencialmente estresantes (Boillos, 2006; Jouper \& Gustafsson, 2013; Olmedilla, 2005).

De acuerdo con los resultados antes descritos se confirmó lo planteado por Olmedilla (2005), quien después de implementar una intervención psicológica en futbolistas, observó cambios favorables (aunque no significativos) en los factores: Control de Estrés, Influencia de la Evaluación del Rendimiento y Habilidad Mental, señalando que estos tres factores evalúan el manejo de diferentes técnicas y el uso de distintas estrategias para un mejor afrontamiento de las demandas deportivas.

Como estrategia para el manejo adecuado del estrés, las técnicas de relajación (como las implementadas en este estudio) ocupan un lugar primordial para la autorregulación psicológica que es requerida durante los diferentes momentos que la práctica del deporte plantea al sujeto que la realiza, por los beneficios directos e indirectos que estas conllevan (Olmedilla, Ortega, Andreu, \& Ortín, 2010). En definitiva, las técnicas de relajación sirven para que el deportista pueda regular de forma consciente y voluntaria el estado de sobreactivación que podría en un momento determinado menoscabar su rendimiento deportivo; la relajación muscular y progresiva se fundamenta en el control y activación del deportista, principalmente en la esfera de lo orgánico (muscular, cardiovascular y respiratoria), así como cognoscitiva (Nieuwenhuys \& Oudejans, 2011).

Contrariamente, los factores Influencia de Evaluación del Rendimiento y Motivación mostraron cambios desfavorables en la post evaluación. Es de llamar la atención que los jugadores lograron cambios positivos en Control de Estrés, en cambio en la variable Influencia de Evaluación del Rendimiento no se obtuvieran cambios en un sentido favorable como se esperaría, ya que las evidencias de otros estudios plantean esa relación. Por ejemplo, Lavarello (2004) en un estudio con N=156 futbolistas, encontró que los jugadores que obtienen puntuaciones más altas en control del estrés van a afrontar mejor la ansiedad generada al ser evaluado socialmente, y por lo tanto con la Influencia de Evaluación del Rendimiento; aunque, también observó una relación directa y significativa con la motivación, afirmando que a mayores niveles de influencia de la evaluación, se favorecen mayores niveles de motivación entre los futbolistas. El mismo Lavarello (2005), señaló la importancia del impacto de situaciones estresantes de la competencia deportiva y la evaluación del mismo por parte de otras personas o del propio deportista. Gimeno, Buceta y Pérez-Llantada 
(2001), definen a la influencia de la evaluación del rendimiento como una variable de estrés específica, asociada con la evaluación y la ansiedad social.

De alguna forma, los resultados negativos de nuestro estudio en cuanto a influencia de la evaluación del rendimiento, así como la baja en motivación podrían explicarse por indicadores como: perder la concentración, manifestación de ansiedad, aparición de dudas de hacerlo bien, o sentirse o no motivado, emisión de juicios por parte del entrenador, los compañeros, los rivales, la prensa, entre otras personas que pueden emitir juicios sobre las ejecuciones de los futbolistas y llegar a influenciar en su rendimiento (Bara, Andrade, Miranda, Núñez, Martín-Albó, \& Ribas, 2011; Corredoira, Rodríguez, \& Sanjiao, 2012).

A manera de conclusión se puede afirmar que esta investigación plantea la posibilidad de mejorar el rendimiento deportivo, evidenciado a partir de resultados útiles con instrumentos de evaluación como el CPRD sobre los diferentes factores psicológicos de los jugadores, que ha servido de base de conocimiento para intervenir exitosamente favoreciendo el rendimiento del equipo. Se sugiere realizar estudios en donde se repliquen los contenidos de la intervención; así mismo, se incremente el número de jugadores participantes, que favorezca a posibilidad de por lo menos contar con dos grupos experimentales de comparación entre las evaluaciones.

\section{Referencias:}

1. Álvarez, O., Falco, C., Estevan, I., Molina-García, J., \& Castillo, I. (2013). Intervención psicológica en un equipo de gimnasia rítmica deportiva: estudio de un caso. Revista de Psicología del Deporte, 22(2), 395-401.2.Bara, F. M., Andrade, D., Miranda, R., Núñez, J. L., Martín-Albó, J., \& Ribas, P. R. (2011).

2. Preliminary validation of a brazilian version of the sport motivation scale. Universitas Psychologica, 10(2), 557-566.

3. Boillos, D. (2006). La mentalización en el fútbol moderno: guía practica para entrenadores y futbolistas. Madrid: Editorial Biblioteca Nueva S.L.

4. Boot W. R., Simons, D. J., \& Stothart, C. (2013). The pervasive problem with placebos in psychology: why active control groups are not sufficient to rule out placebo effects. Perspective Psychology Science, 8(4):445-54. Recuperado de: doi:10.1177/1745691613491271.

5. Brown, D. \& Fletcher, D. (2016). Effects of Psychological and Psychosocial Interventions on Sport Performance: A Meta-Analysis. Sports Medicine, 47, 77-99. Disponible en: https://www.researchgate.net/publication/303694216. 
6. Burtscher, J., Furtner, M., Sachse, P., \& Burtscher, M. (2011). Validation of a German version of the Sport Motivation Scale (SMS28) and motivation analysis in competitive mountain runners. Perceptual \& Motor Skills, 112(3), 807-820.

7. Cachón, J., Rodrigo, M., Campoy, T., Linares, D., \& Zagalaz, M. L. (2012). Fútbol Sala y educación. Aprendizaje de un deporte colectivo para los escolares. Journal of Sport and Health Research, 4(3), 145254.

8. Carmona, J. F. Guzmán, A., \& Olmedilla, A. (2015). Efectos de un programa de formulación de objetivos y moldeamiento del pase en jóvenes jugadores de fútbol. Revista de Psicología del Deporte, 25, 81-88.

9. Carron, A. V., Brawley, L. R., \& Widmeyer, W. N. (1998). Measurement of cohesion in sport and exercise. En J. L. Dude (Ed.), Advances in sport and exercise psychology measurement (pp. 213226). Morgantown, WV: Fitness Information Technology.

10. Carron, A. V., Colman, M. M., Wheeler, J., \& Stevens, D. (2002). Cohesion and performance in sport: A meta-analysis. Journal of Sport \& Exercise Psychology, 24, 168-188.

11. Carron, A. V, Hausenblas, H. A., \& Eys, M. A. (2005). Group Dynamics in Sport. Morgantown, WV: Fitness Information Technology.

12. Castillo-Rodríguez, A. (2011). Aumento del rendimiento físico a través de método ATR en fútbol amateur. Revista Digital. 16 (159). Disponible en http://www.efdeportes.com. Revisado el 16 de enero de 2014.

13. Cerasoli, C. P., Nicklin, J. M., \& Ford, M. T. (2014). Intrinsic motivation and extrinsic incentives jointly predict performance: A 40-year meta-analysis. Psychological Bulletin, 140(4) 1, 9801008. Recuperado de: http://dx.doi.org/10.1037/a0035661

14. Chicau, C. M., Silva, C. M., \& Palmi, J. (2012). Programa de Intervención psicológica para laoptimización del concepto de equipo (team building) en jóvenes futbolistas. Revista de Psicología del Deporte, 21(1), 49-58.

15. Corredoira, F., Rodríguez, F., \& Sanjiao, R. (2012). Características psicológicas del futbolista en Formación mediante el CPRD. Alto Rendimiento, 2 (7), 1-10.

16. Eys, M. A., Loughead, T. M., Bray, S. R., \& Carron, A. V. (2009). Perceptions of cohesion by youth sport participants. The Sport Psychologist, 23, 330- 345. 
17. Fenoy, J. \& Campoy, L. (2012). Rendimiento deportivo, estilos de liderazgo y evitación experiencial en jóvenes futbolistas almerienses. Revista de Psicología del Deporte, 21(1), 137- 142.

18. Fletcher, D. \& Daniels, K. A (2012). Systematic review of stress management interventions with sport performers. Sport Exercises. Performance Psychology, 1, 173-93

19. Garcés de Los Fayos, E. J., De Francisco, C., \& Arce, C. (2012). Inventario de Burnout en Deportistas Revisado (IBD-R). Revista de Psicología del Deporte, 21, 271-278.

20. García-Calvo, T., Leo-Marcos, F.M., Sánchez-Miguel, P.A., Jiménez-Casturera, R., \& Cervelló-Gimeno, E. (2008). Importancia de los aspectos motivacionales sobre el grado de cohesión en equipos de fútbol. Revista de Iberoamericana de Psicología del Ejercicio y el Deporte. 3 (1), 61-74.

21. García, P.H. (2008). Variables psicológicas y rendimiento deportivo en el fútbol profesional. Tesis Doctoral. Madrid

22. García Naveira, A. (2011). Aproximación al empleo profesional del coaching en el deporte. Información Psicológica, 101, 26-39.

23. García, O. \& Serrano, V. (2011). El análisis de la realidad del fútbol desde una perspectiva científica. [Reseña del libro Fútbol e Innovación, de Julen Castellano Paulis (Ed.)]. Revista de Psicología del Deporte, 20(2), 808-813.

24. García-Ucha, F. (2009). Historia y actualidad de la psicología y el deporte en Cuba. Revista Iberoamericana de Psicología del Ejercicio y el Deporte, 4(2), 307-316.

25. Gimeno, F., Buceta, J. \& Pérez Llantada, M. (2001). El cuestionario Características Psicológicas Relacionadas con el Rendimiento Deportivo (CPRD): Características psicométricas. Análise Psicologicas, 1, $93-133$.

26. Godoy-Izquierdo, D., Vélez, M., \& Pradas, F. (2009). Nivel de Dominio de las habilidades Psicológicas en Jóvenes Jugadores de Tenis de Mesa, Bádminton y Fútbol. Revista de Psicología del Deporte. 18 (1), 7-22.

27. González-Campos, G. (2011). Aspectos tácticos y psicopedagógicos a tener en cuenta en el desarrollo deportivo de un partido de fútbol de relevancia institucional. Journal of Sport and Health Research, 3(1), 91- 96.

28. González Campos, G., Campos Mesa, M. C., \& Romero Granados, S. (2014). Análisis de la influencia de la evaluación del rendimiento en jugadores de un equipo de fútbol. Retos. Nuevas Tendencias en Educación Física, Deporte y Recreación, 25, 85-89. 
29. Granero-Gallegos, A. \& Baena-Extremera, A. (2013). Análisis preliminar exploratorio del "Sport Motivation Scale (SMS)" adaptado a la Educación Física. Espiral. Cuadernos del Profesorado, 6(12), 314. Recuperado de: http://www.cepcuevasolula.es/espiral.

30. Jouper, J. \& Gustafsson, H. (2013). Mindful recovery: a case study of a Burned-out elite shooter. The Sport Psychologist, 27, 92-102.

31. Lavarello, J. (2004). Estudio correlacional entre las variables medidas a través del CPRD en futbolistas de las divisiones menores. Revista de Medicina del Deporte Cubana, 3, 18-26.

32. Lavarello, S. J. (2005). Estudio comparativo de los niveles medidos por el cprd entre dos grupos etáreos de las divisiones menores de dos equipos de fútbol de primera división. Efdeportes.com.

33. López-López, I. S. (2011). La evaluación de variables psicológicas relacionadas con el rendimiento en futbol: Habilidades psicológicas para competir y personalidad resistente. Tesis Doctoral No Publicada. Granada. Universidad de Granada.

34. López-López, I. S., Jaenes-Sánchez, J.C., \& Cárdenas-Vélez, D. (2013). Adaptación para futbolistas (CPRD-F) del cuestionario "Características psicológicas relacionadas con el rendimiento deportivo” (CPRD). Cuadernos de Psicología del Deporte, 13 (2), 21-30.

35. Lorenzo, M. \& Bohorquez, M. R. (2014). Análisis de la percepción de los futbolistas acerca del proceso de retirada deportiva. Revista Iberoamericana de Psicología del Ejercicio y del Deporte, 8(1), 107126.

36. Lorenzo, J., Pujals, C., Navarro, R., \& Lorenzo, A. (2012). Análisis de los efectos de un programa de intervención psicológica en jóvenes jugadores de baloncesto. Revista de Psicología del Deporte, 21(1), 43-48.

37. McCormick, A., Meijen, C., \& Marcora, S. (2015). Sports Medicine, 45, 997. Recuperado de: doi:10.1007/s40279-015-03196.

38. McWhirter, J. (2016). Manual del Master en Developmental Behavioural Modelling (DBM®)- Intervención Psicológica con $D B M \AA$ : desde el Desarrollo Profesional al Trabajo con el Cliente. Valencia: Universidad de Valencia.

39. Menéndez-Veliz, F.M. \& Estua-Ampudia, J.E. (2013). Entrenamiento psicológico para contribuir al desarrollo de la preparación psicológica. EFDeportes.com, Revista Digital, 18 (184).

40. Navlet, M. R. (2012). Ansiedad, estrés y estrategias de afrontamiento en el ámbito deportivo: Un estudio centrado en la diferencia entre 
deportes (Tesis Doctoral sin publicar). Universidad Complutense de Madrid, Madrid, España.

41. Nieuwenhuys, A. \& Oudejans, R. (2011). Training with anxiety: short-and long- term effects on police officers' shooting behavior under pressure. Cognitive Processing, 12(3), 277-288.

42. Olmedilla, A. (2005). Factores psicológicos y lesiones en futbolistas. Un estudio correlacional. Murcia: Murcia: Quaderna Editorial.

43. Olmedilla, A., Ortega, E., Ortín, F.J., \& Andreu, M.D. (2010). Entrenamiento Psicológico en Fútbol Base de Élite: Percepción de Aplicabilidad e Índices de Satisfacción. Revista de Iberoamericana de Psicología del Ejercicio y el Deporte, 3 (1), 35-52.

44. Pazo, C. I., Sáenz-López, P. \& Fradua, L. (2012). Influencia del contexto deportivo en la formación de los futbolistas la selección española de fútbol. Revista de Psicología del Deporte, 21(2), 291299.

45. Perry, J. (2015). Examining the roles of motivation and conscientiousness in individual sport performance. USA: Saint Louis University. ProQuest Dissertations Publishing, 1568091. Recuperado de: http://search.proquest.com/docview/1608995955

46. Ramírez, S. (2001). Estandarización y análisis de un instrumento español de psicología deportiva (CPRD: Buceta, Gimeno y Pérez) para una población mexicana.

47. Rumbold. J. L., Fletcher, D., \& Daniels. K. A (2012). Systematic review of stress management interventions with sport performers. Sport Exercises. Performance Psychology, 1, 173-93

48. Sánchez-Sánchez, J., Yagüe, J. M., Fernández, R. C., \& Petisco, C. (2014). Efectos de un entrenamiento con juegos reducidos sobre la técnica y la condición física de jóvenes futbolistas. RICYDE Revista Internacional de Ciencias Del Deporte, 37, 221-234

49. Serra-Olivares, J.; González-Víllora, S., \& García-López, L.M. (2011). Comparación del rendimiento de juego de jugadores de fútbol de 8-9 años en dos juegos modificados 3 contra 3. Revista Cuadernos de Psicología del Deporte. 11 (2), 77-91.

50. Wagstaff, C. R. D. (2014). Emotion regulation and sport performance. Journal of Sport Exercise Psychology, 36(4), 401-12. Recuperado de: doi:10.1123/jsep.2013-0257 